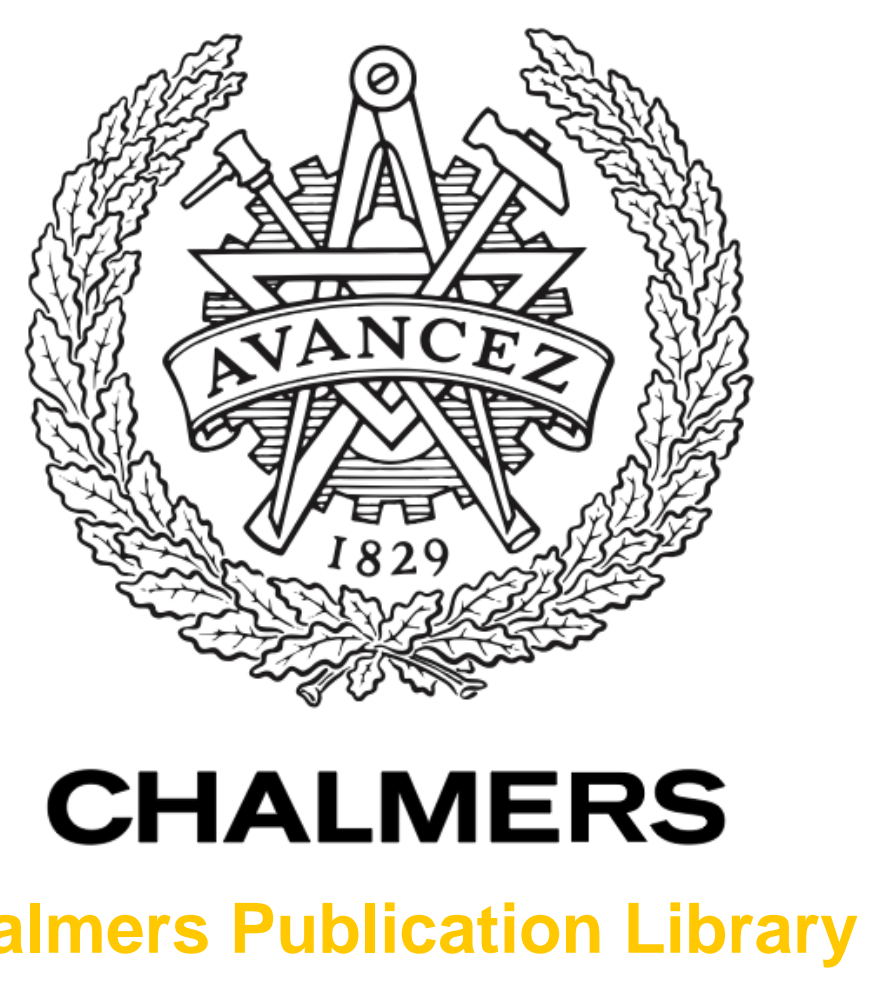

Thrimage: An Empathy-Oriented Discussion Tool for Classroom Use

This document has been downloaded from Chalmers Publication Library (CPL). It is the author's version of a work that was accepted for publication in:

\title{
MobileHCl '15: Proceedings of the 17th International Conference on Human-Computer Interaction with Mobile Devices and Service
}

Citation for the published paper:

Lundgren, S. ; Torgersson, O. ; Björk, S. (2015) "Thrimage: An Empathy-Oriented

Discussion Tool for Classroom Use". MobileHCI '15: Proceedings of the 17th International

Conference on Human-Computer Interaction with Mobile Devices and Service pp. 628-635.

http://dx.doi.org/10.1145/2786567.2792901

Downloaded from: http://publications.lib.chalmers.se/publication/229213

Notice: Changes introduced as a result of publishing processes such as copy-editing and formatting may not be reflected in this document. For a definitive version of this work, please refer to the published source. Please note that access to the published version might require a subscription. 


\section{Thrimage: An Empathy-Oriented Discussion Tool for Classroom Use}

\author{
Sus Lundgren \\ Department of Applied IT \\ Chalmers University of Technology \\ SE-412 96 Gothenburg, Sweden \\ sus.lundgren@chalmers.se

\section{Olof Torgersson} \\ Department of Applied IT \\ University of Gothenburg \\ SE-412 96 Gothenburg, Sweden \\ olof.torgersson@ait.gu.se

\section{Staffan Björk} \\ Department of Applied IT \\ University of Gothenburg \\ SE-412 96 Gothenburg, Sweden \\ staffan.bjork@ait.gu.se
}

Permission to make digital or hard copies of part or all of this work for personal or classroom use is granted without fee provided that copies are not made or distributed for profit or commercial advantage and that copies bear this notice and the full citation on the first page. Copyrights for third-party components of this work must be honored. For all other uses, contact the Owner/Author.

Copyright is held by the owner/author(s)

MobileHCI '15 Adjunct, August 24-27, 2015, Copenhagen, Denmark ACM 978-1-4503-3653-6/15/08.

http://dx.doi.org/10.1145/2786567.2792901

\begin{abstract}
Thrimage is a class-application where pupils choose and rank images in relation to a given word or notion. In seeing who else chose similarly, as well as in a debriefing teacher-led discussion, pupils gain insight in others' way of thinking, and learn to argument for their own opinion but also to respect others, both of which supports the development of empathy and mutual understanding. The design is part of a long-running design exploration on designing of collaborative, colocated experiences using mobile devices, in combination with an educational need.
\end{abstract}

\section{Author Keywords}

Thrimage; image sharing; empathy

\section{ACM Classification Keywords}

H.5.2 [Information Interfaces and Presentation]: User Interfaces.

\section{Introduction}

In late April 2014, the YouTube video Look Up by Gary Turk $^{1}$ went viral, and in a year it has collected $50,394,110$ views. Its topic: how our smartphones alienate us from each other. In response to this, we have formulated a design program entitled Bursting the

\footnotetext{
${ }^{1}$ https://youtu.be/Z7dLU6fk9QY
} 
Mobile Bubble [6] to help open up the design space around this and similar situations, aiming to explore the design of collaborative, co-located experiences using mobile devices - i.e. where technology supports or mediates communication, rather than being the central focus of attention.

We are of course not the only ones taking this direction; e.g. the two applications MobiPhos [2] and MobiComics [3] address this issue; the former by requiring collocation to share and explore pictures, the other by providing co-creation of comics using smart phones and shared public displays. Several games or gamified designs also explore the topic, e.g. giving and following instructions in teams [4]. Some of these experiences may also have an educational character [7], or they may be classified as a 'serious' game, for example to study team coordination in a disaster response scenario [1]. Recently, a framework for designing collocated mobile experiences has been released as well [5].

To explore these issues further, we have recently been designing a series of "bubble-bursting" games within an educational context; one of them, which will be presented here, is an image-based discussion tool called Thrimage.

\section{Context: The MOSAIK-project}

The Swedish National Agency for Education has set up numerous learning goals and knowledge requirements that are to be met during the first nine years of education in Sweden. Of these, many are comparably easy to teach and assess whereas others are more subjective and overarching, e.g. "to be able to imagine and understand the situation other people are in", "to respect others", and "to be able to interact with others, based on knowledge regarding differences and similarities in living conditions, culture, language, religion and history". In a project evolving around images and interpretation of images we set out to provide tools for supporting these goals. One of these designs is Thrimage.

\section{Thrimage at a glance}

Thrimage is a tablet-based game that is to be played simultaneously by all pupils in class, playing the same session. The teacher submits a word that ey ${ }^{2}$ wants to discuss with the class; it should be a complex notion like fear, friendship, respect, sustainability etc. Each pupil then, on their own tablet, chooses and ranks three images (hence the name, Thrimage) from a predefined set of 40 images. When all pupils have chosen, or when the teacher ends the turn, each pupil finds out which other pupil(s) they were the most similar too, counting not only choice of images but which rank they were given. This provides a sense of shared understanding and mutual recognition between them: "Oh, wow, Bera chose very similar to me, how interesting!"

In addition, the teacher gets both a summary on the images chosen by the class, as well as a detailed record on who chose what. Ey can then run a discussion with the class, basing it on the chosen images. "I can see many of you chose the lion. You are one of them, Iyo - can you tell us how you were thinking?" In answering, Iyo knows ey was not the only one choosing that image, which serves to make em more confident in answering.

Through its design, the application acknowledges

2 In line with instructions on gender-neutral language, the gender neutral Spivak pronouns are used 
that people may associate differently depending on their background and by doing so creates a basis for a discussion on these different interpretations. The motivation for this is to allow the pupils to gain a better understanding of others, as well as a better understanding of the given notion.

\section{Design Motivations and Design Process}

Thrimage is loosely based on a casual board game called Compatibility ${ }^{4}$. It is played in teams, and each player has an identical deck of picture cards. Given a target word, the objective of the team members is to select the same images associated with it, and preferably also rank them the same way for a higher score. What players seem to enjoy in this game is not so much the gameplay per se, but the discussions that arise when the selected images are uncovered. "Why did you choose THAT image?" This particular aspect lent itself very well to the stated learning goals (and thus design goals) of understanding others' ways of reasoning.

\section{Compatibility vs Thrimage}

There are three major differences between

Compatibility and Thrimage, however. Firstly, since the idea is fairly simple, we chose to design Thrimage for younger pupils (aged 7-9) whereas Compatibility is a game for adults, featuring images meaningful for adults (e.g. the White House and the stock market; two notions that do not make sense for Swedish eight-yearolds). Secondly, Compatibility is played in teams, with a maximum of eight players, whereas we wanted to

4 Compatibility was designed by Craig Browne in 1996. See https://boardgamegeek.com/boardgame/2604/compatibility for details. involve the whole class, and have them play together, since this would further strengthen the goal of exploring how others felt or reasoned in relation to a certain notion. This decision opened up for the third difference: whereas Compatibility may well be played with physical cards, Thrimage runs on tablets, utilizing computational technology and various algorithms to find matches and other types of statistics.

Iterative Participatory Design as Process Since we were changing both target group and gameplay, we carried out an iterative process as follows:

Preparation: Image Selection: Looking at how the original Compatibility deck of images is composed, a researcher and one of the children selected 108 images.

First iteration: Image Literacy: In four groups of four, the children were shown the total of 108 images. Their initial reactions (i.e. spontaneous questions and comments) were recorded after which we used a structured process - selecting especially interesting images, matching images to words motivating why, and lastly selecting particularly uninteresting images - to try to find out if they preferred certain images or types of images. While this did produce some input for modifying the collection of images, the pupils were very different in image literacy and general maturity, and moreover some sessions were completely skewed by one of the children influencing the others.

Thus, it was hard to draw any clear conclusions. In general, images with people, animals or situations were more favored than passive images of nature, landscapes or everyday objects. Interestingly, images 
that were beforehand believed to be more challenging images - for example images of sculptures and art also sparked a significant interest.

Second Iteration. Teacher Interview: A few rounds of Compatibility were played with three teachers (in separate interviews), and the general full-class version was discussed. All teachers were very much in favor of the design idea and saw a lot of potential use for it in the classroom, since it can be used to discuss complex notions, e.g. what friendship, respect, etc. actually entail. They also commented on how it provided a way

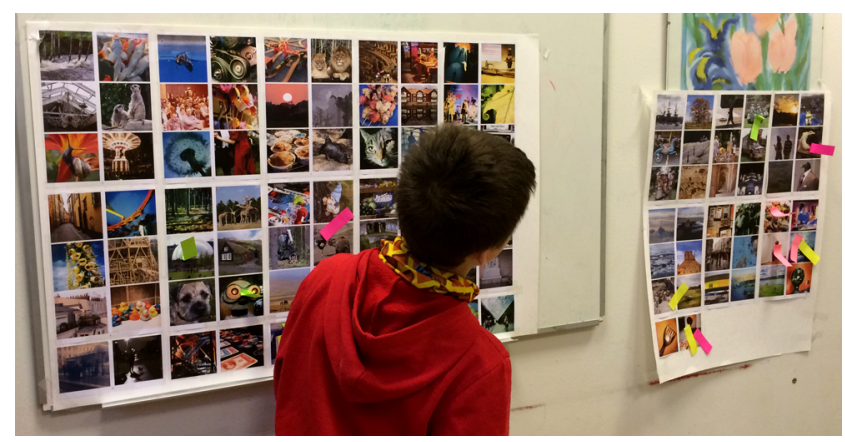

Figure 1: One of the steps in the first iteration was that pupils denoted especially interesting images with Post-Its; here one of the pupils is scrutinizing the selection to see where ey will put eirs.

to practice reasoning and argumentation. One of them - teaching newly arrived refugees and immigrants aged 10 to 15 - also saw it as a tool for language development.

We also discussed what kind of actions and data the teacher-version of the app would entail; for instance it was evident that teachers wanted the ability to provide words/notions themselves.

Third Iteration: Playtest: Using the input from the first phase, 40 images were selected. Most of them came from the original 108, but some new were added to create a varied collection. Out of these we created physical card decks for the pupils.

Two groups of five pupils played a version of the game where the researcher provided a word (e.g. danger, death, fun, speed) and all secretly chose four images and ranked them. Tension and excitement was great when the images were uncovered one by one,

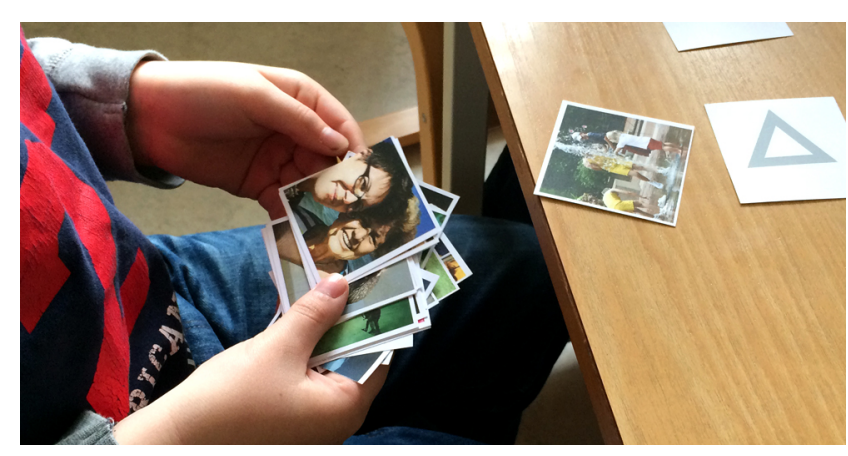

Figure 2: Third iteration: a pupil going through the deck of images to find the ones fitting the given word.

and what pupils found to be the most interesting was to see who had chosen the same images as themselves. This was expected and welcomed; we had been relying on this effect since it creates positive emotions and a sense of mutual understanding. "Look! We have the 
same images! We are SUPERBUDDIES!"

When asked if they could suggest rules however, the pupils faltered. Being similar to someone else was important to them, but they agreed that being alone in choosing a certain image could not be bad either, since that person may have thought of something different. However one of the pupils mentioned something about collecting buddies, which sparked an idea in the researcher running the session.

\section{Fourth Iteration: Interaction design and UI}

design: At this point it was time to pinpoint interaction and certain design choices, so the designer-researcher that had taken part in all the previous phases gathered all data and inspiration collected and turned this into an interaction framework and UI, which was then programmed.

The application was then tested with a small group of children at one of the participating schools. The children had no problems understanding the basic functionality of the app and how to select, order and reorder images. It was noticed that they did not try to view images in full screen mode and accordingly they did not figure out how to do it. It was also noticed that some screens contained too much text.

\section{Upcoming iterations}

The application will be refined in line with the results from the first user test, e.g., there will be less text to read for the children in the GUI. We will then perform a full class game session in both classes to evaluate the application and the learning outcomes.

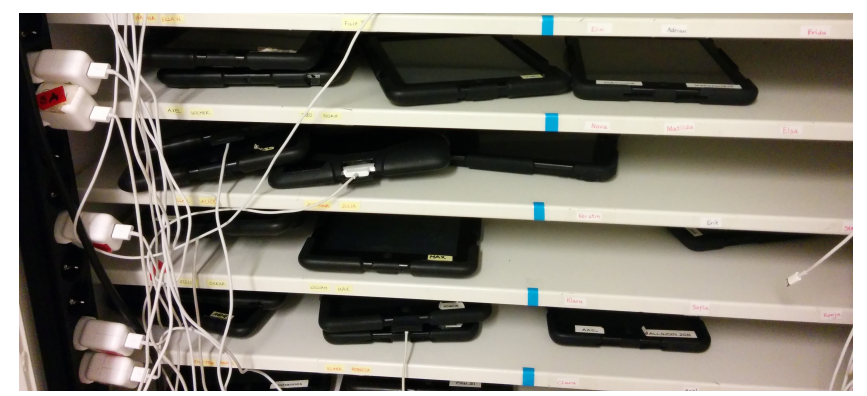

Figure 3: iPad cabinet in school Vällsjöskolan.

\section{Technical Solution}

Thrimage was implemented as a native application for iOS using the Swift programming language. This choice is based on the fact that the iPad dominates the school market strongly in Sweden. In quite a few schools each pupil is equipped with eir own iPad, which is then used as a tool in all subjects. For instance, this is the case in one of the schools participating in the project.

A basic goal in the technical design of Thrimage is to design a standalone tool that can be downloaded and be put to use without any need for registration procedures and user accounts. In the accordance with the aim of designing for co-located interaction the app should also demand that users playing the game are located close to each other. Based on these

requirements Thrimage makes use of Apple's Multipeer Connectivity Framework ${ }^{5}$ to set up the communication between the teachers' and the pupils' iPads. The teacher's tablet functions as a server and advertises a service, which the pupil app automatically connects to

5

https://developer.apple.com/library/ios/documentation/Multipe erConnectivity/Reference/MultipeerConnectivityFramework/ind ex.html\#//apple_ref/doc/uid/TP40013328 
when started. To allow for simultaneous sessions in nearby classrooms the pupils need to enter a session id on their device. While this adds a little bit of extra complexity it is not that easy to avoid if there are multiple nearby sessions.

\section{Thrimage: The design}

Thrimage consists of two distinct interfaces, the teacher interface and the pupil interface.

The Teacher Interface

Here, the teacher starts the session. Ey selects a word that is being sent to the pupils' tablets, and ey can then oversee the status of the pupils: whether they are still choosing, or whether they have submitted their choices. The teacher can end the session whenever ey likes - which of course also gives pupils the possibility to opt out by never choosing any images.

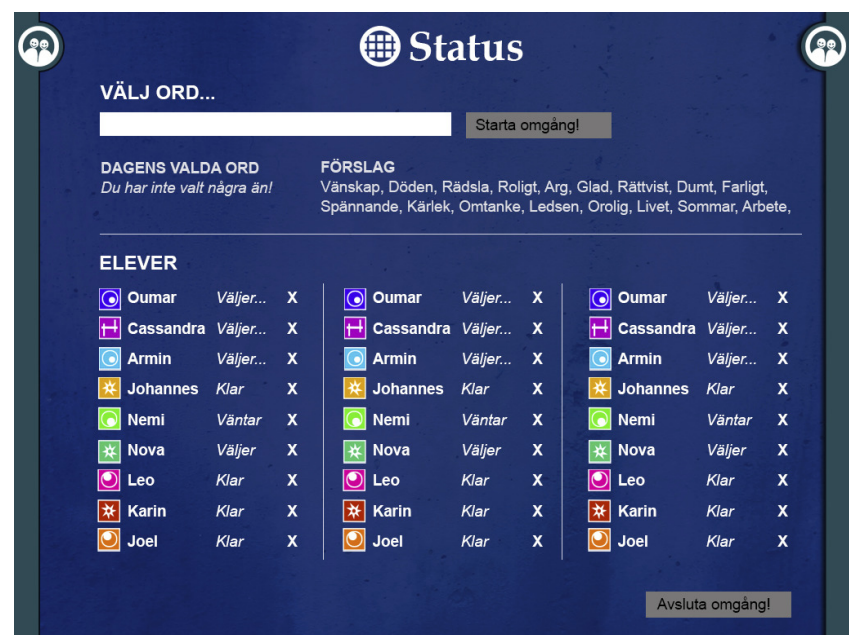

Figure 4: Teacher overview.
Once a session is ended, the teacher has three different entry points to the data. Most interestingly, ey gets an overview on all images chosen, shown as a map of instances. If selecting an image, ey can also see which pupils chose it, and how they ranked it. Lastly, ey can see exactly which images a pupil chose.

\section{The pupil interface}

The pupil interface lets each pupil submit their name and create a personalized icon representing em. The main interface is designed as a circular set of views where one navigates between them by swiping, or using one of the icons present at the top of the screen. The design makes use of the pupils' understanding of basic interaction patterns like swiping, simple taps and using long press to access secondary actions which were observed during the design process.

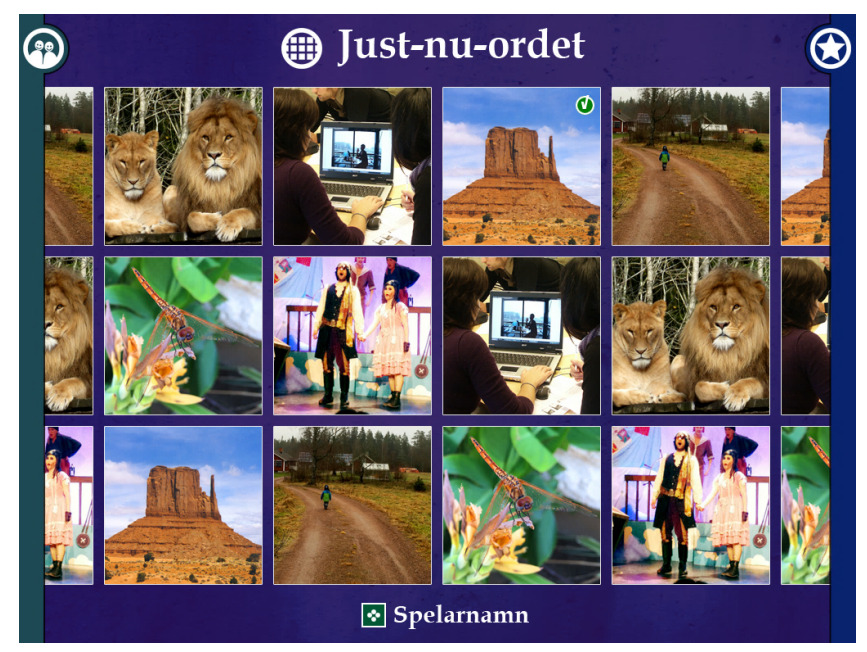

Figure 5: The main view for selecting images. 
When a session starts the main screen for selecting images is presented (see Figure 5). Based on how people tend to proceed when playing Compatilibility and observations made in the second iteration, selecting images is a two-step process where a number of candidate images are selected; this selection is then refined and ordered in the second step as illustrated in Figure 6

When the pupils have made their choice they are presented with an overview showing who made a similar choice and ranking, i.e. who their "superbuddy" is in this particular turn. The intention is to increase the pupils' curiosity for each other and their similarities and differences.

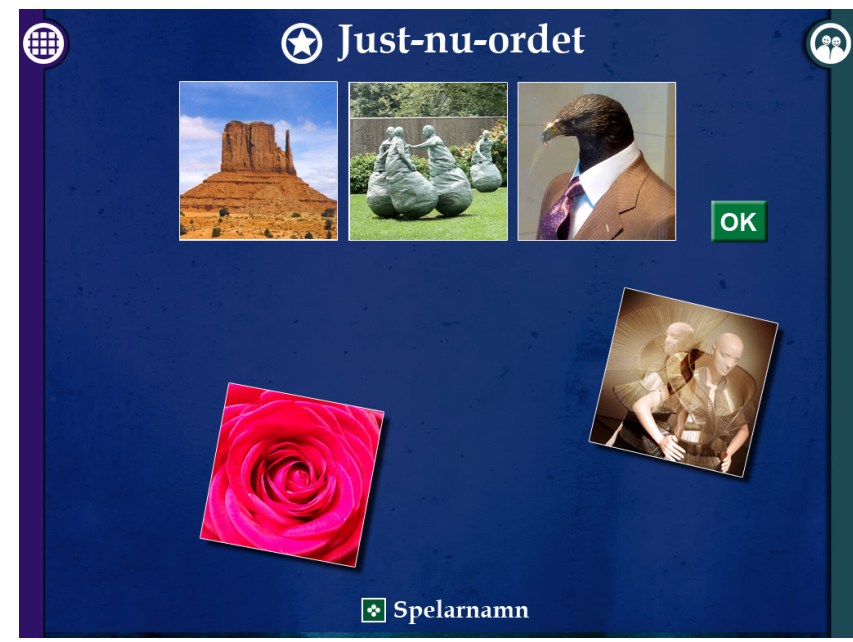

Figure 6: A pupil has selected 3 images, two were left out.
When the teacher ends the turn, they see an overview of all turns played (Figure 7), as well as a list of which class mates they have "collected" as superbuddies insofar, and which ones are left.

The collection of superbuddies serves to gamify the tool somewhat as well as support and highlight the mutual understanding and recognition that occurs when one shares a view with someone unexpected.

Privacy Issues vs Intended Learning Outcomes One issue that was discussed a lot during the development of Thrimage was how much the pupils should see of how other pupils selected their images.

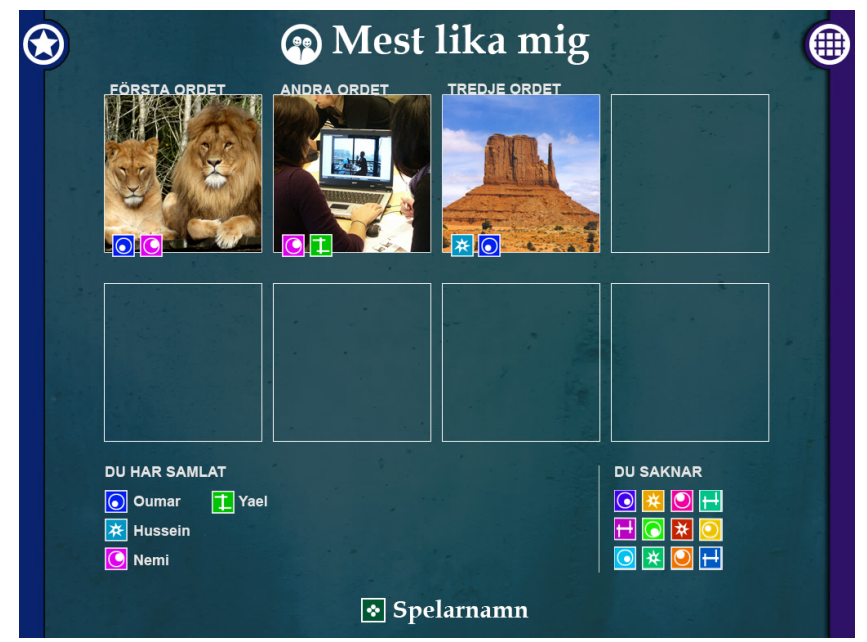

Figure 8: Turn summary, showing which classmates have been "collected" as superbudddies (and in relation to which notion), as well as the icons of the classmates not yet "collected" 
On the one hand the purpose of Thrimage is to let pupils compare their results to those of others in order to reflect upon how they and the others think. On the other hand there is a risk that a pupil that selects images that no one else selected might feel estranged from the group. Moreover, one can image use cases when it may not be appropriate to show superbuddies, e.g. if the word chosen opens up for very personal interpretations and the teacher wants to encourage students to be honest. As a solution to this latter issue, we have provided the teacher with a means to anonymize all selections.

\section{Conclusion}

We have presented the Thrimage application aimed at being an aid in achieving learning goals related empathy and understanding of other people in elementary school in Sweden. The application lets each pupil in a class take part in a shared activity through the use of a combination of a number of communicating devices where the devices are connected using the iOS Multipeer Connectivity Framework.

\section{Acknowledgements}

Thanks to VINNOVA for funding the project as well as to the avid, active and very helpful pupils of class $2 b$ at Rambergsskolan and class 1 at Vällsjöskolan and their teachers. Thanks also to William Gabrielsson for coding.

\section{References}

1. Fischer, J.E., Jiang, W., Kerne, A., Greenhalgh, C., Ramchurn, S.D., Reece, S., Pantidi, N. and Rodden, T. Supporting team coordination on the ground: requirements from a mixed reality game. Proc. COOP '14, Springer (2014), 46-67.

2. Lucero, A., Holopainen, J. and Jokela, T. PassThem-Around: Collaborative Use of Mobile
Phones for Photo Sharing. Proc. of $\mathrm{CHI}$ '11, ACM Press (2011), 1787-1796.

3. Lucero, A., Holopainen, J. and Jokela, T. MobiComics: collaborative use of mobile phones and large displays for public expression. Proc. MobileHCI '12, ACM Press (2012), 383-392.

4. Moran, S., Pantidi, N., Bachour, K., Fischer, J.E., Flintham, M., Rodden, T., Evans, S. and Johnson, S. Team Reactions to Voiced Agent Instructions in a Pervasive Game. Proc. IUI '13. ACM Press (2013), 371-382.

5. Lundgren, S., Fischer, J. E., Reeves, S. and Torgersson, O: Designing Mobile Experiences for Collocated Interaction. Proc of CSCW '15, ACM Press (2015)

6. Lundgren, S., Torgersson, O. Bursting the mobile bubble. First International Workshop on Designing Mobile Face-to-Face Group Interactions, ECSCW'13 (2013)

7. Toups, Z. O. and Kerne, A. 2007. Implicit coordination in firefighting practice: design implications for teaching fire emergency responders. Proc. CHI '07. ACM Press (2007), 707-716. 